\title{
Self-Reported Weather Sensitivity is Associated with Clinical Symptoms and Structural Abnormalities in Patients with Knee Osteoarthritis: A Cross-Sectional Study
}

\author{
Yan Xue · Yan Chen · Ding Jiang · Lin Wang · Xuezong Wang • \\ Ming Li · Yuyun Wu $\cdot$ Min Zhang · Jian Pang · Hongsheng Zhan • \\ Yuxin Zheng $\cdot$ Daofang Ding $\cdot$ Yuelong Cao
}

Received: May 1, 2021 / Accepted: June 17, 2021 / Published online: August 13, 2021

(C) The Author(s) 2021

\section{ABSTRACT}

Introduction: Patients with knee osteoarthritis (KOA) often complain about clinical symptoms affected by weather-related factors. The purpose of the present study was to use cross-sectional analysis to determine whether weather sensitivity was associated with clinical symptoms, as well as structure abnormalities, in KOA patients. Methods: Data from 80 participants were obtained from the Feng Hans Shi Effects on OA (FHS) study, an OA cohort study initiated in

Yan Xue and Yan Chen contributed equally to this work.

Supplementary Information The online version contains supplementary material available at https:// doi.org/10.1007/s40744-021-00340-w.

Y. Xue · Y. Chen · D. Jiang · L. Wang · X. Wang ·

M. Li · Y. Wu · M. Zhang · J. Pang - H. Zhan .

Y. Zheng · Y. Cao $(\bowtie)$

Shi's Center of Orthopedics and Traumatology, Shuguang Hospital Affiliated to Shanghai University of Traditional Chinese Medicine, Shanghai 201203, China

e-mail: ningtcm@126.com

Y. Xue

Shanghai Yangzhi Rehabilitation Hospital (Shanghai Sunshine Rehabilitation Centre), Shanghai 201613, China

\section{Ding}

School of Rehabilitation Science, Shanghai University of Traditional Chinese Medicine, Shanghai 201203, China
China in 2015. The weather sensitivity of each participant was determined by a self-reported questionnaire. The following measurements were used to assess clinical outcomes: Western Ontario and McMaster Universities Osteoarthritis Index (WOMAC) for symptoms, and semi-quantitative Whole-Organ Magnetic Resonance Imaging Score (WORMS) for cartilage defects and marrow abnormalities of magnetic resonance imaging (MRI). Chi-square with Cochran-Armitage test for trend and regression analysis were used to evaluate the associations between weather sensitivity and WOMAC and WORMS of KOA patients.

Results: Most of the KOA participants (57.5\%) perceived the weather as affecting their kneejoint clinical symptoms. After adjusting for age, gender, and body mass index (BMI), weather sensitivity was not only associated with knee pain $[\mathrm{OR}=3.3(95 \%$ CI $1.1,9.9), P=0.032]$, dysfunction $[\mathrm{OR}=5.5 \quad(95 \%$ CI $1.8,16.8)$, $P=0.003]$, and overall clinical symptoms [OR = 3.3 (95\% CI 1.1, 10.2), $P=0.034$ ], but also associated with cartilage defect $[\mathrm{OR}=3.1(95 \%$ CI 1.1, 8.5), $P=0.027$ ] and marrow abnormality $[\mathrm{OR}=3.0$ (95\% CI 1.1, 8.1), $P=0.029$ ].

Conclusions: In KOA patients, weather sensitivity was associated with clinical symptoms and structural abnormalities. Future longitudinal study is warranted for the causal relationship. 


\section{Infographic:}

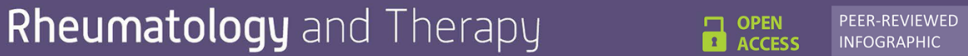

\section{Self-Reported Weather Sensitivity is Associated with Clinical Symptoms and Structural Abnormalities in Patients with Knee Osteoarthritis: A Cross-Sectional Study}

\section{Xue, Y., Chen, Y, Jiang, D. et al. Rheumatol Ther (2021). https://doi.org/10.1007/s40744-021-00340-w}
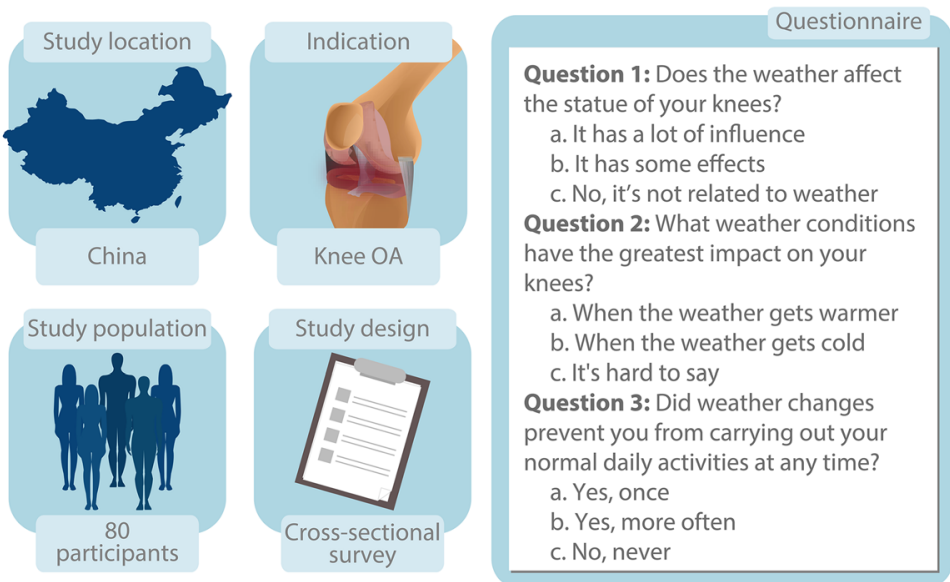

Question 1: Does the weather affect statue of your knees?

b. It has some effects Question 2: What weather conditions

participants survey

b. Yes, more often

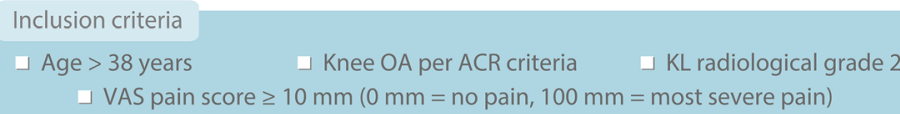

\section{Why carry out this study?}

People with knee OA frequently report that their clinical symptoms - including pain, stiffness, and disability - are sensitive to weather.

$O A$ is a complex disease for which subjective weather sensitivity has not been fully investigated.

This study was conducted to determine whether self-reported weather sensitivity was associated with clinical symptoms and structural abnormalities in patients with knee OA via a cross-sectional survey.

\section{What was learned from the study?}

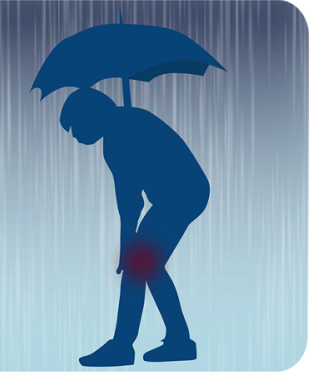

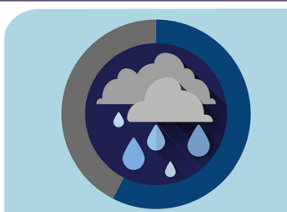

$57.5 \%$

of participants reported weather sensitivity
After adjusting for age, sex, and BMI, weather sensitivity was associated with:

$$
\begin{aligned}
& \text { Clinical } \\
& \text { symptoms }
\end{aligned}
$$

Knee pain

Dysfunction

Overall clinical symptoms
Structural abnormalities

\section{Cartilage} defects

Marrow abnormality

A longitudinal study is warranted to determine whether causal relationships exist.

Abbreviations: $\mathrm{OA}=$ Osteoarthritis; $\mathrm{ACR}=$ American College of Rheumatology; $\mathrm{KL}=$ Kellgren-Lawrence; VAS = Visual analogue scale; BMI = body mass index

$\triangle$ Adis This infographic represents the opinic 
Keywords: Knee osteoarthritis (KOA); Weather sensitivity; McMaster Universities Osteoarthritis Index (WOMAC); Whole-Organ Magnetic Resonance Imaging Score (WORMS)

\section{Key Summary Points}

Knee osteoarthritis (KOA) is a multifactorially disease.

People with KOA frequently report that their clinical symptoms were sensitive to weather.

The purpose of the study was to determine the association between self-reported weather sensitivity and clinical symptoms as well as structural abnormalities in a cross-sectional design.

The results show that weather sensitivity present was independently associated with knee pain, cartilage defect, and marrow abnormality.

\section{DIGITAL FEATURES}

This article is published with digital features, including an infographic, to facilitate understanding of the article. To view digital features for this article go to https://doi.org/10.6084/ m9.figshare.15135216.

\section{INTRODUCTION}

Knee osteoarthritis (KOA) is a very complicated pathophysiologic joint disease and one of the main causes of disability worldwide [1-3]. The main characteristics of KOA are knee cartilage wear, subchondral bone sclerosis, and deformation of the surrounding bone. KOA patients often experience joint pain and joint movement limitations, as well as other symptoms [4-6].
People with KOA frequently report weather conditions that affect the clinical symptoms of their knees, including pain, stiffness, and disability [7]. Furthermore, many patients with OA feel that they are sensitive to the weather, with changes in temperature affecting their symptoms [8]. Several studies have explored the influence of weather parameters on KOA. For example, research on the association between perceived weather change and $\mathrm{OA}$ has been conducted. Desirée et al. [9] assessed the correlation between the surrounding weather conditions and clinical symptoms of patients with OA in the Netherlands. Timmermans et al. [7] also investigated differences in perception of the effects of weather on joint pain among elderly people with OA in six European countries under different weather conditions. Brennan et al. [10] explored whether weather (in)stability has a greater impact on joint structures and pain perception in individuals from southern Europe. However, all of these studies emphasized objective and external weather parameters rather than subjective and internal feelings from patients. Given that $\mathrm{OA}$ is a multifactorial disease with a more individualized treatment approach, and subjective perception of temperature is often not consistent with actual changes in temperature, it is necessary to evaluate the influence of self-perceived weather sensitivity on KOA. Moreover, the relationship of weather sensitivity to clinical symptoms and structural abnormalities has not been fully investigated, and remains unclear.

Therefore, the purpose of this study was to determine whether weather sensitivity from subjective feelings had an association with clinical symptoms and structural abnormalities in KOA patients in a cross-sectional design.

\section{METHODS}

\section{Design and Study Samples}

Our study population comprised a subsample of participants of the Feng Hans Shi Effects on OA (FHS) study, which was a multicenter, prospective, cohort study focusing on KOA, from July 2015 to July 2017, at three clinical centers. In 
this study, $80 \mathrm{KOA}$ patients were randomly selected from one of the sub-centers. This study was registered at the Chinese Clinical Trial Registry (ChiCTR1800017777). All participants were interviewed and examined by trained researchers in hospital outpatient departments via related questionnaires and instruments.

The inclusion criteria were as follows: (1) older than 38 years; (2) diagnosis of primary KOA according to the American College of Rheumatology criteria (ACR)[9]; (3) Kellgren-Lawrence (KL) radiological grade 2[11]; and (4) visual analogue scale (VAS) pain score $\geq 10 \mathrm{~mm} \quad(0 \mathrm{~mm}=$ no pain, $100 \mathrm{~mm}=$ most severe pain). Participants were excluded from the study if they met any of the following exclusion criteria at the beginning of, or during, the study: (1) rheumatoid arthritis; (2) serious cardiovascular or kidney diseases; (3) severe KOA and recently proposed to take kneejoint replacement surgery; (4) magnetic resonance imaging (MRI) contraindications such as the installation of pacemakers, artificial metal valves, or cornea/artery retention.

\section{Self-Perceived Weather Sensitivity}

To assess self-perceived weather sensitivity, participates were asked to answer a questionnaire concerning weather sensitivity, which included three questions, each with three types of answers. These questions were also referred to published questionnaire concerning weather sensitivity (Table S1) [10].

Patients who chose option A or B for any of the three questions were classified as weathersensitive patients, while those who chose option $\mathrm{C}$ for all three questions were classified as non-weather-sensitive patients.

\section{Assessment of Osteoarthritis Symptoms}

The clinical characteristics included basic demographic data and the Western Ontario and McMaster Universities Osteoarthritis Index (WOMAC), which measures knee pain (five items), stiffness (two items), and function (17 items) over the past $48 \mathrm{~h}$. The traditional 24-item WOMAC is scored using a VAS ranging from "none" to "most severe" (the total range is from 0 to 240; higher scores indicate worse KOA symptoms) [12]. The VAS was used to assess knee pain for initial participant screening.

\section{MRI Acquisition and Assessment of Knee Structure}

Participants underwent MRI of the knees using the same superconducting magnetic resonance scanners (1.5 Tesla, Achieva, Philips, The Netherlands). Imaging sequences included the following: sagittal proton density (PD)-weighted fast spin-echo (FSE) (TR $4800 \mathrm{~ms}$, TE $35 \mathrm{~ms}$, 3-mm slice thickness, 0-mm interslice gap, 32 slices, $288 \times 192$ matrix, 2 NEX, 140-mm 2 FOV, 8 ETL); axial T2-weighted (TR $4680 \mathrm{~ms}$, TE $13 \mathrm{~ms}, 3-\mathrm{mm}$ slice thickness, 0-mm interslice gap, 20 slices, $288 \times 192$ matrix, 2 NEX, 140-mm 2 FOV, 8 ETL); and coronal PD-FSE (TR $6650 \mathrm{~ms}$, TE $15 \mathrm{~ms}$, TI $100 \mathrm{~ms}, 3-\mathrm{mm}$ slice thickness, $0-\mathrm{mm}$ interslice gap, 28 slices, $256 \times 192$ matrix, 2 NEX, 140-mm 2 FOV, 8 ETL).

Cartilage and bone marrow lesions were assessed using the semi-quantitative WholeOrgan Magnetic Resonance Imaging Score (WORMS) method [13] for KOA. The assessment of cartilage defects was based on 14 regions, which were made up of the medial and lateral compartments of the tibia (anterior, central, and posterior), femur (anterior, central, and posterior), and patella. Bone marrow lesions were graded in the 14 regions mentioned above, as well as in the region of the tibia beneath the tibial spine.

Cartilage signal and morphology were scored with an eight-point scale, as follows: $0=$ normal thickness and signal; $1=$ normal thickness but increased signal on T2-weighted images; 2.0 = partial-thickness focal defect $<1 \mathrm{~cm}$ in greatest width; $2.5=$ full-thickness focal defect $<1 \mathrm{~cm}$ in greatest width; $3=$ multiple areas of partial-thickness (Grade 2.0) defects intermixed with areas of normal thickness, or a Grade 2.0 defect wider than $1 \mathrm{~cm}$ but $<75 \%$ of the region; $4=$ diffuse ( $\geq 75 \%$ of the region) partial-thickness loss; $5=$ multiple areas of fullthickness loss (grade 2.5) or a grade-2.5 lesion 
Table 1 Characteristics of the KOA participants according to weather sensitivity

\begin{tabular}{llll}
\hline Characteristic & $\begin{array}{l}\text { Non-weather-sensitive group } \\
(\boldsymbol{n}=\mathbf{3 4})\end{array}$ & $\begin{array}{l}\text { Weather-sensitive group } \\
(\boldsymbol{n}=\mathbf{4 6})\end{array}$ & $\boldsymbol{P}$ value \\
\hline Age (years) & $61.1(1.1)$ & $61.8(0.9)$ & 0.638 \\
Gender $(\%$ female) & $21(35)$ & $39(65)$ & $\mathbf{0 . 0 1 9}^{\mathbf{a}}$ \\
Height $(\mathrm{cm})$ & $162.7(1.3)$ & $161.7(1.0)$ & 0.538 \\
Weight $(\mathrm{kg})$ & $63.3(1.8)$ & $63.6(1.2)$ & 0.883 \\
BMI $\left(\mathrm{kg} / \mathrm{m}^{2}\right)$ & $23.8(0.5)$ & $24.4(0.5)$ & 0.427 \\
KOA duration (months) & $24.2(1.3)$ & $22.2(1.1)$ & 0.264 \\
VAS pain $(0-100 \mathrm{~mm})$ & $26.1(1.4)$ & $30.0(1.5)$ & 0.085 \\
\hline
\end{tabular}

Bold value indicate the $P<0.05$

Results displayed as mean (SEM) unless otherwise indicated, $P$ values for independent $t$ tests unless otherwise indicated $B M I$ body mass index, $K O A$ knee osteoarthritis, $V A S$ visual analogue scale/score

${ }^{\text {a }}$ Pearson Chi-squared test

wider than $1 \mathrm{~cm}$ but $<75 \%$ of the region; $6=$ diffuse $(\geq 75 \%$ of the region) full-thickness loss.

Subarticular bone marrow abnormality was defined as poorly marginated areas of increased signal intensity in the normally fatty epiphyseal marrow on PD-weighted FSE images. The following four-point grading scale was used: $0=$ none; $1=$ less than $25 \%$ of the region; $2=25-50 \%$ of the region; $3=$ greater than $50 \%$ of the region.

All images were scored by two orthopedists with over 10 years of clinical experience who were blinded to each patient's personal information. WORMS of 30 randomly selected subjects were then scored independently by them to determine intra- and inter-rater reproducibility. Intraclass correlation coefficients (ICCs) for intra- and inter-reader agreement of the WORMS cartilage scores were 0.973 (95\% CI $0.944,0.987)$ and 0.986 (95\% CI 0.971, 0.993), respectively. For WORMS marrow abnormality scores, intra-reader ICC was $0.972(95 \%$ CI $0.942,0.986)$ and inter-reader ICC was 0.986 (95\% CI 0.970, 0.993).

\section{Statistical Analysis}

Differences in characteristics between weathersensitive and non-weather-sensitive KOA participants were examined with independent sample $t$-tests for continuous data (except WOMAC and WORMS) and Chi-square tests for categorical data.

According to the quartile of all participants in the cross-sectional analysis, WOMAC and WORMS were converted into four levels of categorical variables by interquartile range for further analysis. The differences in WOMAC and WORMS between weather-sensitive and nonweather-sensitive participants were first tested using Pearson's Chi-square tests and Fisher's exact tests. Cochran-Armitage tests for linear trends were also conducted for variables that showed statistically significant relationships following Chi-square tests. In addition, a Mann-Whitney test was used to compare the WORMS between the weather-sensitive group and non-weather-sensitive group.

Binary logistic regression analysis was applied to estimate the association of weather sensitivity with WOMAC and WORMS scores. When included in the regression analysis, originally discrete variables of them were converted into binary dependent factors via the median, while the presence or absence of weather sensitivity was used as an independent factor. All models were adjusted for age, gender, and body mass index (BMI).

All statistical analyses were performed in IBM SPSS Statistics (version 17.0) and R (version 
Table 2 Comparison of KOA participants with and without weather sensitivity in WOMAC

\begin{tabular}{|c|c|c|c|c|c|c|c|}
\hline WOMAC & & $\begin{array}{l}\text { Non- } \\
\text { weather- } \\
\text { sensitive } \\
\text { group } \\
(n=34)\end{array}$ & $\begin{array}{l}\text { Weather- } \\
\text { sensitive } \\
\text { group } \\
(n=46)\end{array}$ & $\begin{array}{l}\text { Total } \\
\text { participants } \\
(n=80)\end{array}$ & $\chi^{2}$ & $\begin{array}{l}P \text { for independence } \\
\text { (Pearson's Chi- } \\
\text { square test) }\end{array}$ & $\begin{array}{l}P \text { for trend } \\
\text { (Cochran-Armitage } \\
\text { trend tests) }\end{array}$ \\
\hline \multirow{4}{*}{$\begin{array}{l}\text { Pain score } \\
\qquad(0-50)\end{array}$} & $\leq 5.5$ & $14(41.2 \%)$ & $6(13 \%)$ & $20(25 \%)$ & 10.539 & 0.014 & $<0.001$ \\
\hline & $5.5-10.6$ & $9(26.5 \%)$ & $\begin{array}{l}11 \\
\quad(23.9 \%)\end{array}$ & $20(25 \%)$ & & & \\
\hline & $10.6-19.5$ & 7 (20.6\%) & $\begin{array}{l}14 \\
\quad(30.4 \%)\end{array}$ & $21(26.3 \%)$ & & & \\
\hline & $>19.5$ & $4(11.8 \%)$ & $\begin{array}{l}15 \\
\quad(32.6 \%)\end{array}$ & $19(23.8 \%)$ & & & \\
\hline \multirow{4}{*}{$\begin{array}{l}\text { Stiffness } \\
\text { score } \\
\quad(0-20)\end{array}$} & $\leq 0.9$ & $15(44.1 \%)$ & $5(10.9 \%)$ & $20(25 \%)$ & 17.401 & 0.001 & 0.003 \\
\hline & $0.9-4.5$ & $5(14.7 \%)$ & $\begin{array}{l}16 \\
(34.8 \%)\end{array}$ & $21(26.3 \%)$ & & & \\
\hline & $4.5-9.0$ & $11(32.4 \%)$ & $\begin{array}{l}10 \\
\quad(21.7 \%)\end{array}$ & $21(26.3 \%)$ & & & \\
\hline & $>9.0$ & $3(8.8 \%)$ & $\begin{array}{l}15 \\
\quad(32.6 \%)\end{array}$ & $18(22.5 \%)$ & & & \\
\hline \multirow{4}{*}{$\begin{array}{l}\text { Function } \\
\text { score } \\
(0-170)\end{array}$} & $\leq 16.9$ & $15(44.1 \%)$ & $5(10.9 \%)$ & $20(25 \%)$ & 15.754 & 0.001 & $<0.001$ \\
\hline & $16.9-31.1$ & $10(29.4 \%)$ & $\begin{array}{l}10 \\
\quad(21.7 \%)\end{array}$ & $20(25 \%)$ & & & \\
\hline & $31.1-61.5$ & $5(14.7 \%)$ & $\begin{array}{l}15 \\
\quad(32.6 \%)\end{array}$ & $20(25 \%)$ & & & \\
\hline & $>61.5$ & $4(11.8 \%)$ & $\begin{array}{l}16 \\
(34.8 \%)\end{array}$ & $20(25 \%)$ & & & \\
\hline \multirow{4}{*}{$\begin{array}{l}\text { Total } \\
\text { score } \\
(0-240)\end{array}$} & $\leq 26.9$ & $15(44.1 \%)$ & $5(10.9 \%)$ & $20(25 \%)$ & 13.299 & 0.004 & $<0.001$ \\
\hline & $26.9-51.7$ & $8(23.5 \%)$ & $\begin{array}{l}12 \\
(26.1 \%)\end{array}$ & $20(25 \%)$ & & & \\
\hline & $51.7-87.0$ & 7 (20.6\%) & $\begin{array}{l}13 \\
\quad(28.3 \%)\end{array}$ & $20(25 \%)$ & & & \\
\hline & $>87.0$ & $4(11.8 \%)$ & $\begin{array}{l}16 \\
(34.8 \%)\end{array}$ & $20(25 \%)$ & & & \\
\hline
\end{tabular}

Bold value indicates the $P<0.05$

WOMAC Western Ontario and McMaster University Osteoarthritis Index 
Table 3 Comparison of KOA participants with and without weather sensitivity in WORMS

\begin{tabular}{|c|c|c|c|c|c|c|c|}
\hline WORMS & & $\begin{array}{l}\text { Non- } \\
\text { weather- } \\
\text { sensitive } \\
\text { group } \\
(n=33)\end{array}$ & $\begin{array}{l}\text { Weather- } \\
\text { sensitive } \\
\text { group } \\
(n=44)\end{array}$ & $\begin{array}{l}\text { Total } \\
\text { participants } \\
(n=77)\end{array}$ & $\chi^{2}$ & $\begin{array}{l}P \text { for } \\
\text { independence } \\
\text { (Pearson's Chi- } \\
\text { square test) }\end{array}$ & $\begin{array}{l}P \text { for trend } \\
\text { (Cochran-Armitage } \\
\text { trend tests) }\end{array}$ \\
\hline \multirow{4}{*}{$\begin{array}{l}\text { Cartilage score } \\
\qquad(0-84)\end{array}$} & $\leq 10.5$ & $12(36.4 \%)$ & $8(18.2 \%)$ & $20(26 \%)$ & \multirow[t]{4}{*}{7.959} & \multirow[t]{4}{*}{0.047} & \multirow[t]{4}{*}{0.015} \\
\hline & $10.5-15.0$ & $11(33.3 \%)$ & $9(20.5 \%)$ & $20(26 \%)$ & & & \\
\hline & $15.0-20.0$ & $4(12.1 \%)$ & $\begin{array}{l}15 \\
(34.1 \%)\end{array}$ & $19(24.7 \%)$ & & & \\
\hline & $>20.0$ & $6(18.2 \%)$ & $\begin{array}{l}12 \\
(27.3 \%)\end{array}$ & $18(23.4 \%)$ & & & \\
\hline \multirow{4}{*}{$\begin{array}{l}\text { Marrow } \\
\text { abnormality } \\
\text { score }(0-45)\end{array}$} & 0.0 & $22(66.7 \%)$ & $\begin{array}{l}14 \\
\quad(31.8 \%)\end{array}$ & $36(46.8 \%)$ & \multirow[t]{4}{*}{10.256} & \multirow[t]{4}{*}{$0.014^{a}$} & \multirow[t]{4}{*}{0.009} \\
\hline & 1.0 & $1(3.0 \%)$ & $4(9.1 \%)$ & $5(6.5 \%)$ & & & \\
\hline & $1.0-5.0$ & $5(15.2 \%)$ & $\begin{array}{l}18 \\
\quad(40.9 \%)\end{array}$ & $23(29.9 \%)$ & & & \\
\hline & $>5.0$ & $5(15.2 \%)$ & $8(18.2 \%)$ & $13(16.9 \%)$ & & & \\
\hline
\end{tabular}

Bold value indicates the $P<0.05$

WORMS Whole-Organ Magnetic Resonance Imaging Score

a Fisher's exact test

3.6.1). Two-tailed tests set at $P<0.05$ were used to determine statistical significance.

\section{RESULTS}

\section{Participant Characteristics}

A total of 80 subjects $(75 \%$ women) were recruited. Of those, $34(42.5 \%)$ reported no weather sensitivity, whereas $46(57.5 \%)$ did report having weather sensitivity. A total of $96 \%$ of the participants $(n=77$, three patients did not undergo examinations) acquired MRI of the knees. The average age was 61.5 years. The mean ( \pm standard of the mean [SEM]) values for height, weight, BMI, KOA duration, and VAS pain were as follows: $162.1( \pm 0.8) \mathrm{cm}, 63.5$ $( \pm 1.0) \mathrm{kg}, 24.1( \pm 0.3) \mathrm{kg} / \mathrm{m}^{2}, 23.1( \pm 0.9)$ months, and $28.3( \pm 1.1) \mathrm{mm}$, respectively. The characteristics of non-weather-sensitive and weather-sensitive participants are presented in Table 1 . There were no significant differences in demographic factors between the two groups, with the exception that there was a higher proportion of women (65\%) in the weathersensitive group.

\section{Relation of Weather Sensitivity and WOMAC}

The data showed significant differences in WOMAC pain scores $\left[\chi^{2}(3, n=80)=10.539\right.$; $P=0.014], \quad$ stiffness scores $\left[\chi^{2} \quad(3\right.$, $n=80)=17.401 ; P=0.001]$, function scores $\left[\chi^{2}\right.$ $(3, n=80)=15.754 ; P=0.001]$, and total scores $\left[\chi^{2}(3, n=80)=13.299 ; P=0.004\right]$ in the ratio of quartile distribution between non-weathersensitive and weather-sensitive participants (Table 2). The Cochran-Armitage tests for linear trends suggested that higher WOMAC pain $(P<0.001)$, stiffness $(P=0.003)$, function $(P<0.001)$, and total scores $(P<0.001)$ were all associated with weather sensitivity (Table 2 ). 


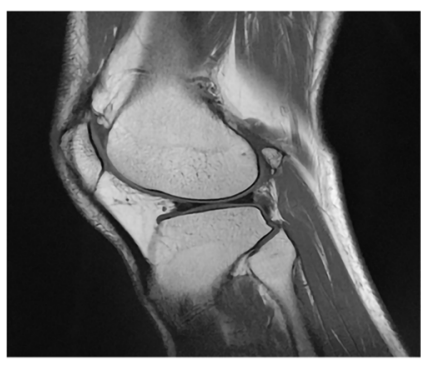

A Non-weather-sensitive group

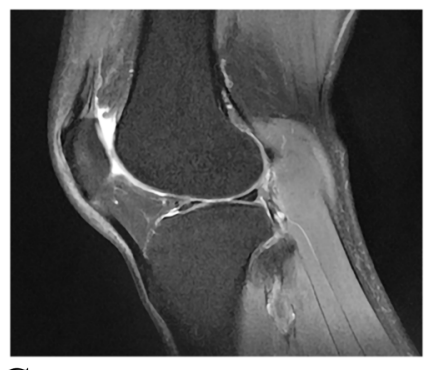

C Non-weather-sensitive group

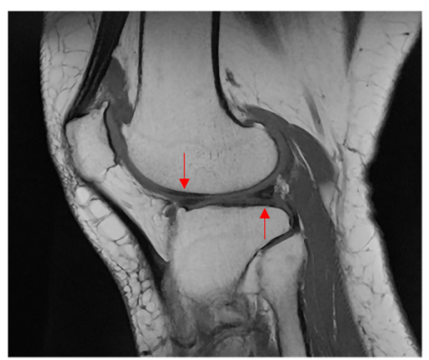

Weather-sensitive group

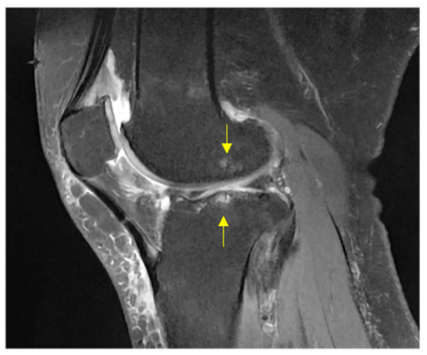

Weather-sensitive group

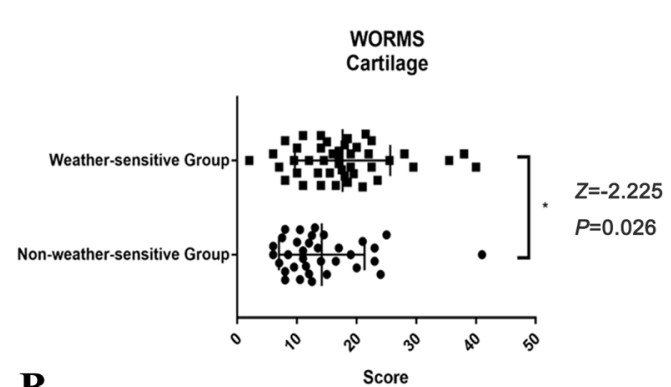

B

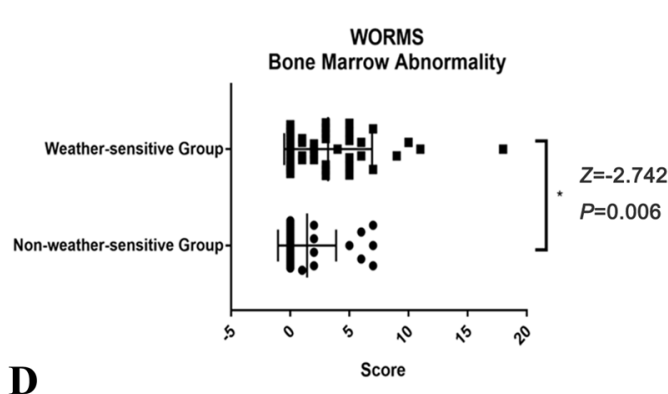

Fig. 1 Comparison of KOA participants with and without weather sensitivity in Cartilage and Marrow abnormality. A Magnetic resonance images (SAG T1weighted FSE) of two groups. Red arrowheads show cartilage loss of the lateral tibia and femur. B The cartilage defects were obviously increased in weather-sensitive group

\section{Relation of Weather Sensitivity and WORMS}

With regard to the comparison of non-weathersensitive and weather-sensitive group, significant differences were tested for the ratio of quartile distribution of WORMS cartilage $\left[\chi^{2}(3\right.$, $n=77)=7.959 ; P=0.047]$ and marrow abnormality scores $\left[\chi^{2}(3, n=77)=10.256 ; P=0.014\right]$ (Table 3$)$. In overall value, WORMS cartilage $(Z=-2.225, \quad P=0.026)$ and marrow abnormality scores $(Z=-2.742, P=0.006)$ of the weather-sensitive group were higher than those of the non-weather-sensitive group (Fig. 1). Furthermore, analysis for trends indicated that WORMS cartilage $(P=0.015)$ and marrow abnormality scores $(P=0.009)$ were both associated with weather sensitivity (Table 3 ). $\left({ }^{*} P<0.05\right)$. C Magnetic resonance images (SAG PD FSE) of two groups. Yellow arrowheads showed bone marrow abnormality of the medial tibia and femur. D The marrow abnormalities were obviously increased in weather-sensitive group $\left({ }^{*} P<0.05\right)$

\section{Association of Weather Sensitivity with WOMAC and WORMS}

Binary logistic regression analysis revealed that weather sensitivity present was independently associated with severe knee pain [OR = $3.6(95 \%$ CI 1.4, 9.1), $P=0.008$ ], severe knee dysfunction $[\mathrm{OR}=5.7(95 \%$ CI 2.2, 15.3), $P<0.001]$, and severe overall clinical symptoms of knee [OR = 3.6 (95\% CI 1.4, 9.1), $P=0.008$ ) (Table 4). After adjusting for age, gender, and BMI, we found that weather-sensitive participants were more likely to have severe knee pain [OR $=3.3(95 \%$ CI 1.1, 9.9), $P=0.032$ ], severe knee dysfunction $[\mathrm{OR}=5.5$ (95\% CI 1.8, 16.8), $P=0.003]$, and severe overall clinical symptoms of knee [OR = 3.3 (95\% CI 1.1, 10.2), $P=0.034$ ] (Table 4).

Univariably, weather sensitivity was independently related to cartilage defect $[\mathrm{OR}=3.7$ (95\% CI 1.4, 9.5), $P=0.008]$ and marrow abnormality $[\mathrm{OR}=3.3 \quad(95 \%$ CI $1.3,8.6)$, $P=0.014]$ (Table 4). After adjusting for age, 
Table 4 Associations of weather sensitivity present with WOMAC and WORMS

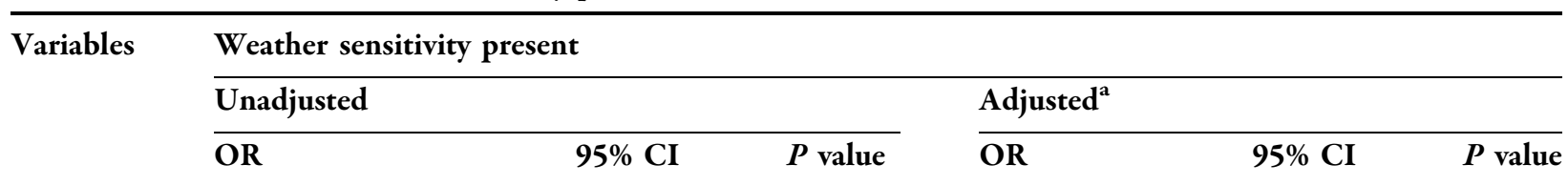

WOMAC

Degree of knee pain

\begin{tabular}{|c|c|c|c|c|c|c|}
\hline Mild & 1.0 [Reference] & & & 1.0 [Reference] & & \\
\hline Severe & 3.6 & $(1.4-9.1)$ & 0.008 & 3.3 & $(1.1-9.9)$ & 0.032 \\
\hline \multicolumn{7}{|c|}{ Degree of knee stiffness } \\
\hline Mild & 1.0 [Reference] & & & $1.0[$ Reference $]$ & & \\
\hline Severe & 1.7 & $(0.7-4.2)$ & 0.245 & 1.1 & $(0.4-3.0)$ & 0.900 \\
\hline
\end{tabular}

Degree of knee dysfunction

$\begin{array}{lllllll}\text { Mild } & 1.0 \text { [Reference] } & & 1.0 \text { [Reference] } & \\ \text { Severe } & 5.7 & (2.2-15.3) & <\mathbf{0 . 0 0 1} & 5.5 & (1.8-16.8) & \mathbf{0 . 0 0 3}\end{array}$

Degree of overall clinical symptoms of knee

\begin{tabular}{|c|c|c|c|c|c|c|}
\hline Mild & 1.0 [Reference] & & & 1.0 [Reference] & & \\
\hline Severe & 3.6 & $(1.4-9.1)$ & 0.008 & 3.3 & $(1.1-10.2)$ & 0.034 \\
\hline
\end{tabular}

WORMS

Degree of the cartilage defect

\begin{tabular}{|c|c|c|c|c|c|c|}
\hline Mild & $1.0[$ Reference $]$ & & & $1.0[$ Reference $]$ & & \\
\hline Severe & 3.7 & $(1.4-9.5)$ & 0.008 & 3.1 & $(1.1-8.5)$ & 0.027 \\
\hline \multicolumn{7}{|c|}{ Degree of marrow abnormality } \\
\hline Mild & $1.0[$ Reference $]$ & & & 1.0 [Reference] & & \\
\hline Severe & 3.3 & $(1.3-8.6)$ & 0.014 & 3.0 & $(1.1-8.1)$ & 0.029 \\
\hline
\end{tabular}

Bold value indicates the $P<0.05$

Independent variable: weather sensitivity present; Dependent variable: degree of knee clinical symptoms and structural abnormalities (dichotomized by the median). Mild: the value is less than the median; Severe: the value is more than the median

$C I$ confidence interval, $O R$ odds ratio

${ }^{\text {a }}$ Adjusted for age, gender, BMI

gender, and BMI, the weather-sensitive participants tended to have severe cartilage defect $[\mathrm{OR}=3.1(95 \% \mathrm{CI} 1.1,8.5), P=0.027]$ and severe marrow abnormality [OR $=3.0(95 \% \mathrm{CI}$ $1.1,8.1), P=0.029$ ] (Table 4).

For all the variables that entered the regression analysis (not shown), the variance inflation factor (VIF) for quantifying the severity of multiple collinearities was between 1.013 and 1.104 (WOMAC formulation) and 1.018 and 1.117 (WORMS formulation), indicating that there was no statistical caveat caused by the strong correlation between the explanatory variables. 


\section{DISCUSSION}

To our knowledge, the current study was the first to investigate the association between selfreported weather sensitivity and clinical symptoms and structural abnormalities assessed using MRI in KOA patients.

KOA is a chronic disease with high heterogeneity, and patients' clinical symptoms often fluctuate and recur. As a small sample study, the current study needed to avoid as much bias as possible in order to ensure the reliability of the results. Therefore, the cases included in the study were all symptomatic KOA patients (VAS pain score $\geq 10 \mathrm{~mm}$ ) with generally mild radiographic features (KL grade $=2$ ).

There were significant composition ratio differences in WOMAC scores between the participants with or without weather-sensitivity, and the Cochrane-Armitage test indicated a trend across different WOMAC levels. Collectively, KOA patients in the weather-sensitive group were more likely to have higher WOMAC scores. After the correction of confunders, weather sensitivity was found to have an independent association with knee pain, dysfunction, and overall clinical symptoms. In our results, there was a significant difference in WOMAC pain scores between the weather-sensitive and weather-insensitive groups, however, difference in VAS scores was not significant. The possible reason is that VAS method is relatively more concise, whereas the WOMAC pain score with five items refining specific evaluation components may reflect patients' conditions more accurately and reliably $[12,14]$.

As a multifactorial disease, the clinical symptoms of KOA may be altered by external factors, such as environmental and climatic factors [15-17]. We found that there was a strong correlation between weather sensitivity and knee pain and dysfunction. This result may have been due to weather-sensitive patients being more vulnerable to external climate change, thus aggravating the symptoms associated with KOA. Despite a previous study that reported weather was not associated with pain [18], a number of studies have confirmed that weather factors are inextricably linked to knee pain $[7,8,17,19-21]$. In a randomized placebocontrolled trial of glucosamine, weather conditions were found to influence pain in OA patients [8]. A 2-week clinical study in European KOA participants also showed that weathersensitive individuals suffered more joint pain than did non-weather-sensitive individuals [7]. Another study has also reported that weather can affect OA symptoms of pain and disability [22].

In a study of Russian clinical samples, climatic factors were found to affect the radiological severity of OA patients [23]. Due to the limitations of radiography, MRI has been identified by the Osteoarthritis Research Society International (OARSI) as the most appropriate imaging modality to assess joint status in OA research studies [24]. We thus used WORMS scores to evaluate and reflect joint structure. In our present study, weather sensitivity was related to the cartilage and marrow abnormalities, with a proportion of patients in the weathersensitive group coinciding with the increased scores of WORMS. By adjusting the covariance, weather sensitivity was also independently associated with knee cartilage defects and bone marrow abnormalities. Related studies have found that the weather may affect the contraction of different tissues in the affected joint and lower temperatures may lead to increased viscosity of synovial fluid in the joint cavity, which may cause joint pain and stiffness $[15,16]$. In addition, weather changes can also lead to early changes in bones and joints [25]. It is possible that weather-sensitive patients are more likely to be affected by weather factors than are non-weather-sensitive patients, implying a potential physiological mechanism for joint degeneration in OA [7].

Previous studies performed in the United States, Australia, and Europe have primarily focused on objective weather parameters rather than individual perceptions of weather, such as barometric pressure, ambient temperature and relative humidity $[8,20]$. With the increasing importance of patient subjective feeling in clinical practice, patient-reported outcome had been appeared more importantly, and had been approved by OARSI as one of clinical trials recommendations [26]. Since the influence of 
weather on the human body varies across individuals, we focused on analyzing subjective selfperception of weather in KOA patients in our present study. Furthermore, by targeting weather reflection of interindividual differences in subjective experiences of pain, using quantitive joint structural assessment, we also obtained objective evaluations of the relationship of self-perceived weather sensitivity with cartilage defects and bone marrow abnormalities in KOA patients.

Our present study also had some potential limitations. Firstly, we only included cross-sectional analyses. The relatively small sample size of our study is also a limiting factor. Secondly, our questionnaire on weather conditions only referred to temperature changes, whereas other weather factors, such as precipitation, atmospheric pressure, humidity, and wind speed, were not considered [27]. Therefore, association between weather sensitivity and symptomatic or structural features of the knee joint may be biased by those factors above. Thirdly, as few studies examined the correlation between weather sensitivity and osteoarthritis clinical symptoms, the reliability and validity examination of the conducted instrument were lack. Pain, as a subjective experience, is usually influenced by complex interactions of biological, psychological, and social factors [28]. It is partly possible that the associations detected between the questions posed to determine the sensitivity to changes in temperature with $\mathrm{OA}$ damage are spuriously due to the global component of pain. Indeed, future studies testing the performance of the instrument using to detect changes in the pain by the weather would further enhance validity of the scale. Most importantly, more longitudinal studies are warranted to further clarify the causal relationship between weather sensitivity and KOA.

\section{CONCLUSIONS}

The current results imply the associations of weather sensitivity and clinical symptoms and structural degradations in KOA patients. Weather-sensitive individuals may be more likely to exhibit severe knee pain, dysfunction, as well as cartilage defects and marrow abnormalities, however, the causal relationship between weather sensitivity and $\mathrm{OA}$ is still unclear.

\section{ACKNOWLEDGEMENTS}

We thank the participants of the study.

Funding. This study was supported by the National Natural Science Foundation of China (81973874; 81373665), the Summit Plateau Team Project in Traumatology of Shanghai University of TCM, Shanghai chronic musculoskeletal disease clinical medical research center (20mc1920600). The journal's Rapid Service Fee was funded by the authors.

Authorship. All named authors meet the International Committee of Medical Journal Editors (ICMJE) criteria for authorship for this article, take responsibility for the integrity of the work as a whole, and have given their approval for this version to be published.

Author contributions. Yan Xue, Yan Chen, and Yuelong Cao have contributed to the design of the study and the analysis of data. Ding Jiang, Lin Wang, Xuezong Wang, and Ming Li have contributed to the recruitment of research subjects and data acquisition. Yuyun Wu, Min Zhang, Jian Pang, Hongsheng Zhan, Yuxin Zheng, and Daofang Ding have contributed to the revision of the manuscript. All the authors read and approved the final version of the manuscript.

Disclosures. Yan Xue, Yan Chen, Ding Jiang, Lin Wang, Xuezong Wang, Ming Li, Yuyun Wu, Min Zhang, Jian Pang, Hongsheng Zhan, Yuxin Zheng, Daofang Ding, and Yuelong Cao declare that they have no conflicts of interest.

Compliance with Ethics Guidelines. This study, registered at the Chinese Clinical Trial Registry (ChiCTR1800017777), was approved by the research ethics committee of Shuguang Hospital affiliated with Shanghai University of 
Traditional Chinese Medicine (approval number: 2013-296-65-01) and performed in accordance with the Helsinki Declaration of 1964, and its later amendments. Informed consent was obtained from each participant included in the present study.

Data Availability. The datasets generated during and/or analyzed during the current study are available from the corresponding author on reasonable request.

Open Access. This article is licensed under a Creative Commons Attribution-NonCommercial 4.0 International License, which permits any non-commercial use, sharing, adaptation, distribution and reproduction in any medium or format, as long as you give appropriate credit to the original author(s) and the source, provide a link to the Creative Commons licence, and indicate if changes were made. The images or other third party material in this article are included in the article's Creative Commons licence, unless indicated otherwise in a credit line to the material. If material is not included in the article's Creative Commons licence and your intended use is not permitted by statutory regulation or exceeds the permitted use, you will need to obtain permission directly from the copyright holder. To view a copy of this licence, visit http://creativecommons.org/licenses/by$\mathrm{nc} / 4.0 /$.

\section{REFERENCES}

1. Palazzo C, Nguyen C, Lefevre-Colau MM, Rannou F, Poiraudeau S. Risk factors and burden of osteoarthritis. Ann Phys Rehabil Med. 2016;59(3): 134-8.

2. Bijlsma JW, Berenbaum F, Lafeber FP. Osteoarthritis: an update with relevance for clinical practice. Lancet. 2011;377(9783):2115-26.

3. Yang M, Jiang L, Wang Q, Chen H, Xu G. Traditional Chinese medicine for knee osteoarthritis: an overview of systematic review. PLoS One. 2017;12(12): e0189884.

4. Farr Ii J, Miller LE, Block JE. Quality of life in patients with knee osteoarthritis: a commentary on nonsurgical and surgical treatments. Open Orthop J. 2013;7:619-23.

5. de Rooij M, van der Leeden M, Heymans MW, Holla JF, Hakkinen A, Lems WF, Roorda LD, Veenhof C, Sanchez-Ramirez DC, de Vet HC, et al. Prognosis of pain and physical functioning in patients with knee osteoarthritis: a systematic review and meta-analysis. Arthritis Care Res (Hoboken). 2016;68(4): 481-92.

6. Muraki S, Akune T, Oka H, En-yo Y, Yoshida M, Saika A, Suzuki T, Yoshida H, Ishibashi H, Tokimura $\mathrm{F}$, et al. Association of radiographic and symptomatic knee osteoarthritis with health-related quality of life in a population-based cohort study in Japan: the ROAD study. Osteoarthritis Cartilage. 2010;18(9):1227-34.

7. Timmermans EJ, van der Pas S, Schaap LA, SanchezMartinez M, Zambon S, Peter R, Pedersen NL, Dennison EM, Denkinger M, Castell MV, et al. Selfperceived weather sensitivity and joint pain in older people with osteoarthritis in six European countries: results from the European Project on OSteoArthritis (EPOSA). BMC Musculoskelet Disord. $2014 ; 15: 66$.

8. McAlindon T, Formica $\mathrm{M}$, Schmid $\mathrm{CH}$, Fletcher J. Changes in barometric pressure and ambient temperature influence osteoarthritis pain. Am J Med. 2007;120(5):429-34.

9. Altman RD. Classification of disease: osteoarthritis. Semin Arthritis Rheum. 1991;20(6 Suppl 2):40-7.

10. von Mackensen S, Hoeppe P, Maarouf A, Tourigny $\mathrm{P}$, Nowak D. Prevalence of weather sensitivity in Germany and Canada. Int $\mathrm{J}$ Biometeorol. 2005;49(3):156-66.

11. Kellgren JH, Lawrence JS. Radiological assessment of osteo-arthrosis. Ann Rheum Dis. 1957;16(4): 494-502.

12. Bellamy N, Buchanan WW, Goldsmith $\mathrm{CH}$, Campbell J, Stitt LW. Validation study of WOMAC: a health status instrument for measuring clinically important patient relevant outcomes to antirheumatic drug therapy in patients with osteoarthritis of the hip or knee. J Rheumatol. 1988;15(12):1833-40.

13. Peterfy CG, Guermazi A, Zaim S, Tirman PF, Miaux Y, White D, Kothari M, Lu Y, Fye K, Zhao S, et al. Whole-Organ Magnetic Resonance Imaging Score (WORMS) of the knee in osteoarthritis. Osteoarthritis Cartilage. 2004;12(3):177-90.

14. Nadrian H, Moghimi N, Nadrian E, Moradzadeh R, Bahmanpour K, Iranpour A, Bellamy N. Validity and reliability of the Persian versions of WOMAC 
Osteoarthritis Index and Lequesne Algofunctional Index. Clin Rheumatol. 2012;31(7):1097-102.

15. Quick DC. Joint pain and weather. A critical review of the literature. Minn Med. 1997;80(3):25-9.

16. Jamison RN, Anderson KO, Slater MA. Weather changes and pain: perceived influence of local climate on pain complaint in chronic pain patients. Pain. 1995;61(2):309-15.

17. Brennan SA, Harney T, Queally JM, O'Connor McGoona J, Gormley IC, Shannon FJ. Influence of weather variables on pain severity in end-stage osteoarthritis. Int Orthop. 2012;36(3):643-6.

18. Wilder FV, Hall BJ, Barrett JP. Osteoarthritis pain and weather. Rheumatology (Oxford). 2003;42(8): 955-8.

19. Timmermans EJ, Schaap LA, Herbolsheimer F, Dennison EM, Maggi S, Pedersen NL, Castell MV, Denkinger MD, Edwards MH, Limongi $\mathrm{F}$, et al. The influence of weather conditions on joint pain in older people with Osteoarthritis: results from the European Project on OSteoArthritis. J Rheumatol. 2015;42(10):1885-92.

20. Ferreira ML, Zhang Y, Metcalf B, Makovey J, Bennell KL, March L, Hunter DJ. The influence of weather on the risk of pain exacerbation in patients with knee osteoarthritis-a case-crossover study. Osteoarthritis Cartilage. 2016;24(12):2042-7.

21. Peultier L, Lion A, Chary-Valckenaere I, Loeuille D, Zhang Z, Rat AC, Gueguen R, Paysant J, Perrin PP. Influence of meteorological elements on balance control and pain in patients with symptomatic knee osteoarthritis. Int J Biometeorol. 2017;61(5): 903-10.
22. Dorleijn DM, Luijsterburg PA, Burdorf A, Rozendaal RM, Verhaar JA, Bos PK, Bierma-Zeinstra SM. Associations between weather conditions and clinical symptoms in patients with hip osteoarthritis: a 2-year cohort study. Pain. 2014;155(4):808-13.

23. Kalichman L, Korosteshevsky M, Batsevich V, Kobyliansky E. Climate is associated with prevalence and severity of radiographic hand osteoarthritis. Homo. 2011;62(4):280-7.

24. Hunter DJ, Altman RD, Cicuttini F, Crema MD, Duryea J, Eckstein F, Guermazi A, Kijowski R, Link TM, Martel-Pelletier J, et al. OARSI Clinical Trials Recommendations: knee imaging in clinical trials in osteoarthritis. Osteoarthritis Cartilage. 2015;23(5):698-715.

25. Belkin V, Livshits G, Otremski I, Kobyliansky E. Aging bone score and climatic factors. Am J Phys Anthropol. 1998;106(3):349-59.

26. McAlindon TE, Driban JB, Henrotin Y, Hunter DJ, Jiang GL, Skou ST, Wang S, Schnitzer T. OARSI Clinical Trials Recommendations: design, conduct, and reporting of clinical trials for knee osteoarthritis. Osteoarthritis Cartilage. 2015;23(5): 747-60.

27. Timmermans EJ, van der Pas S, Dennison EM, Maggi S, Peter R, Castell MV, Pedersen NL, Denkinger MD, Edwards $\mathrm{MH}$, Limongi $\mathrm{F}$, et al. The influence of weather conditions on outdoor physical activity among older people with and without Osteoarthritis in 6 European Countries. J Phys Act Health. 2016;13(12):1385-95.

28. Cascella M, Thompson NS, Muzio MR, Forte CA, Cuomo A. The underestimated role of psychological and rehabilitation approaches for management of cancer pain. A brief commentary. Recenti Prog Med. 2016;107(8):418-21. 